\title{
Le système éducatif chinois
}

\section{Kechao Xing}

\section{OpenEdition}

\section{Journals}

Édition électronique

URL : https://journals.openedition.org/ries/1755

DOI : 10.4000/ries. 1755

ISSN : 2261-4265

\section{Éditeur}

France Education international

\section{Édition imprimée}

Date de publication : 1 avril 2004

Pagination : 129-137

ISBN : 978-2-85420-560-2

ISSN : 1254-4590

\section{Référence électronique}

Kechao Xing, "Le système éducatif chinois ", Revue internationale d'éducation de Sèvres [En ligne], 35 | avril 2004, mis en ligne le 23 novembre 2011, consulté le 05 juillet 2021. URL : http:// journals.openedition.org/ries/1755; DOI : https://doi.org/10.4000/ries.1755

Ce document a été généré automatiquement le 5 juillet 2021

(c) Tous droits réservés 


\title{
Le système éducatif chinois
}

\author{
Kechao Xing
}

1 En préalable à toute présentation du système éducatif chinois, il est indispensable d'avoir en mémoire les notions suivantes sur la Chine :

- une civilisation de plus de cinq mille ans ;

- une population d'au moins un milliard trois cents millions (soit dix-neuf à vingt fois plus que la France) composée de cinquante-six ethnies très différentes au sens le plus large du terme, dont les Hans représentent l'écrasante majorité (plus de $90 \%$ ) ;

- une superficie de 9,6 millions de $\mathrm{km}^{2}$ égale à celle du Canada ou de l'Europe ;

- une écriture idéographique peut-être unique au monde composée de plus de quatre-vingt mille caractères, dont quatre mille les plus usuels, minimum que les jeunes doivent connaître et utiliser au cours de leurs premières années d'études pour pouvoir en commencer d'autres ensuite ;

- après une longue histoire prospère et glorieuse, cent ans sous l'humiliation et même l'occupation étrangère (1840-1949) puis l'indépendance depuis 1949;

- une économie en cours de rapide développement mais avec des disparités régionales considérables, de l'est à l'ouest, des villes à la campagne, du centre aux frontières, à croire qu'il existe plusieurs Chine ;

-à la fin du XXe siècle, vingt-cinq étudiants pour dix mille habitants, soit $10 \%$ des jeunes entre dix-huit et vingt-quatre ans.

\section{Héritage des millénaires}

2 L'enseignement scolaire existe en Chine depuis plus de trois mille ans. On trouvait déjà des écoles officielles dans la dynastie des Zhou, au $\mathrm{XI}^{\mathrm{e}}$ siècle avant J.-C. L'épanouissement des écoles privées pendant la période Printemps et Automne, au VII siècle avant notre ère, avec la dégradation de la dynastie, ouvrit une nouvelle voie.

Pendant de très longues années, l'enseignement scolaire fut réservé à une poignée de privilégiés, d'où le paradoxe qu'un écolier pouvait être un, sinon le grand intellectuel du village pour en devenir plus tard l'instituteur, après l'échec au concours de sélection des fonctionnaires que les Occidentaux appellent mandarins. 
Malgré son caractère initialement pratique (six arts étaient enseignés : la politesse, la danse et la musique, le tir à la flèche, la conduite des chevaux et des chars, la lecture et la composition, le calcul) et sa grande souplesse - devenant plus tard disparité en ce qui concerne le choix d'un enseignement de vocabulaire, de morale, de connaissances sociales et scientifiques, ou de littérature -, le contenu resta, pendant très longtemps, lié au confucianisme sur le plan idéologique et au concours de sélection des fonctionnaires sur le plan institutionnel.

5 Ainsi, l'enseignement traditionnel chinois était :

- fortement lié au keju (concours de sélection des fonctionnaires) qui dura mille trois cents ans (du VII au XXe siècle);

- très socialisé et moralisé, voire politisé, en raison de l'adoption du confucianisme comme principe dominant ;

- fermé presque totalement aux femmes ;

- limité presque exclusivement à l'interprétation et à l'explication des classiques, d'où des insuffisances évidentes dans le domaine scientifique, et notamment technologique, malgré les quatre grandes inventions (boussole, papier, art d'imprimer et poudre explosive);

- fondé sur la mémorisation comme méthode principale d'apprentissage ;

- soumis à l'autorité presque absolue du maître.

\section{Un siècle de tâtonnements}

6 En 1904, la loi de réforme promulguée par Guangxu, avant-dernier empereur chinois, créa l'école nouvelle en abolissant le keju et en introduisant le système éducatif occidental dans l'Empire moribond pour le rendre plus dynamique. Depuis, l'enseignement en Chine, y compris en ce qui concerne les établissements scolaires, se rapproche de plus en plus de celui des autres pays.

7 Pendant un demi-siècle de bouleversements sociaux, de guerres et même d'occupation étrangère, le système occidental, y compris son contenu moderne, s'est enfin installé en Chine avec cependant un énorme contraste entre ville et campagne: de très bonnes universités d'un côté et une situation d'analphabétisme presque totale de l'autre.

8 Après la fondation de la République populaire de Chine en 1949, des expériences motivées par de bonnes intentions, mais fortement influencées par les idées gauchistes, ne purent aboutir au résultat espéré : la création d'un système éducatif scientifique moderne et propre à la Chine. Ce furent, au début des années cinquante, la nationalisation des établissements, l'apprentissage auprès de l'ex-Union soviétique pendant les onze premières années de la République et dix années de Révolution culturelle - jusqu'à la destruction totale de l'éducation nationale.

9 La politique de réforme et d'ouverture, en 1978, donna une nouvelle vie à l'éducation en Chine : plusieurs règlements utiles et nécessaires furent établis (ou rétablis), dont le concours d'entrée à l'université et le système de grades universitaires.

10 La promulgation, en 1986, de la loi dite « Obligation scolaire» (de neuf ans) constitua un nouveau jalon pour le développement général de l'éducation. En moins de dix ans, l'enseignement obligatoire fut généralisé à travers le pays et le taux de scolarisation est aujourd'hui supérieur à $97 \%$. 


\section{Structure et administration}

\section{Le préscolaire}

11 Quoique non-obligatoire, la préscolarisation des enfants est de plus en plus souvent observée en raison de l'élévation du niveau de vie et de l'application de la politique de l'enfant unique. Dans les villes, les bons jardins d'enfants sont très bien équipés et très recherchés, tandis que dans les zones rurales, c'est encore la famille qui se charge le plus souvent de cet enseignement. Les jardins d'enfants sont gérés sectoriellement et recrutent des enseignantes diplômées des écoles normales de l'enseignement préscolaire (au niveau de l'enseignement secondaire du second cycle, c'est-à-dire trois ou quatre années après le collège).

\section{La scolarité obligatoire}

Les enfants chinois, quelque soit leur sexe, leur ethnie et leur origine sociale, ont le droit, à l'âge de six ans, de suivre la scolarité obligatoire de neuf ans qui se répartit, selon les régions, entre cinq ou six années pour l'enseignement élémentaire et trois ou quatre années pour l'enseignement secondaire du premier cycle.

13 L'école primaire relève de la compétence du secteur (quartier dans les villes, commune à la campagne) et choisit ses enseignants parmi les diplômés des écoles normales de même niveau que les enseignantes des jardins d'enfants; le collège est géré au niveau de l'arrondissement dans les villes, du district à la campagne et a pour enseignants les diplômés du cycle court de l'enseignement supérieur (trois années après l'examen de fin d'études secondaires). Étant obligatoires, ni l'école primaire ni le collège ne pratiquent plus l'examen d'entrée et, hormis les établissements ou classes expérimentaux, c'est un accès « sectorisé ».

\section{L'enseignement secondaire du second cycle}

14 D'une durée de trois ans, il est assuré au lycée par les diplômés du cycle long de l'enseignement supérieur (quatre années après l'examen de fin d'études secondaires), dont la plupart sont issus des universités dites "normales ». À côté des lycées d'enseignement général, il existe des lycées professionnels et des écoles secondaires spécialisées. À l'exception des établissements pilote et de ceux qui relèvent des secteurs autres que l'Éducation nationale, la gestion de ce niveau est la même que celle de l'enseignement secondaire du premier cycle. Cependant, la grande différence réside dans le concours d'entrée, qui se fait au niveau provincial, pour un second cycle qui n'a plus de caractère obligatoire désormais.

\section{L'enseignement supérieur}

L'enseignement supérieur chinois se caractérise par son recrutement sélectif, son organisation rigide, sa disciplinarisation étroite et son administration complexe. Le concours d'entrée est national et organisé aux mêmes dates chaque année ${ }^{1}$ par les provinces après l'examen de fin d'études secondaires. Le taux de réussite, vu l'inégalité des ressources, varie de $70 \%$ à Beijing, la capitale, à $4 \%$ à Guizhou, une province 
lointaine et montagneuse du sud-ouest. Une fois à l'université, les étudiants n'ont que peu de choix et suivent systématiquement un cursus quasiment uniforme pour obtenir le diplôme et terminer leurs études au même moment. Influencés par le modèle soviétique, les facultés et départements se spécialisent dans des disciplines relativement étroites et le tronc commun de l'enseignement d'ordre général, bien considéré dans de nombreux pays, est peu important en terme de place au sein du système comme en nombre d'heures. Les établissements d'enseignement supérieur ont des réseaux d'administration et de gestion très variés qui peuvent être, verticalement, le district, la province et l'État, et horizontalement les départements gouvernementaux et différents secteurs de la société.

Hormis le cycle court qui décerne seulement un diplôme, les niveaux supérieurs se terminent tous par un grade : licence après quatre ans, maîtrise trois années après la licence, doctorat trois à quatre années après la maîtrise. L'accès à chaque étape est conditionné par la réussite à un concours national. Il n'y a pas de formation « normale " pour les enseignants du supérieur qui sont, pour la plupart, des diplômés $\mathrm{du}$ troisième cycle universitaire, souvent docteurs.

\section{Les manuels scolaires}

17 Ceux qui concernent l'enseignement obligatoire surtout sont rédigés et publiés par des maisons d'édition accréditées mais selon le programme national tout en intégrant une petite partie de contenu «local». Les universités utilisent pour l'essentiel les livres choisis par les professeurs ainsi que les manuels proposés par le ministère, notamment pour le tronc commun général et disciplinaire.

\section{Changements actuels}

18 "Il faut dix ans pour cultiver un arbre et cent ans un homme ", dit un dicton chinois. C'est justement cette caractéristique de longue haleine dans le domaine de l'éducation qui rend moins évidents les changements et les transformations en la matière, notamment par rapport à la politique et à l'économie. Pourtant, nombreux sont ceux, Chinois et étrangers, qui ont constaté une évolution nette de l'éducation en Chine au cours de ces dernières années, parallèlement à son remarquable essor économique.

\section{Augmentation des investissements}

19 «Un pays pauvre en face d'une demande très importante en éducation », telle est la situation chinoise. Malgré les lois et règlements gouvernementaux décrétant depuis dix ans l'augmentation des investissements dans l'éducation, la croissance est toujours très en dessous de l'exigence de développement. Au lieu de contribuer au développement économique et social, cela l'a sinon empêché, du moins retardé.

20 Le tournant date de 1998, date à laquelle le gouvernement chinois décide d'augmenter la part de l'éducation dans le budget national de $1 \%$ chaque année jusqu'en 2002 et demande aux autorités locales d'en faire autant selon leurs possibilités. Grâce à cette mesure, la part de l'éducation dans le $9^{e}$ plan quinquennal (1996-2000) représente 2,48 fois celle du $8^{\mathrm{e}}$ plan. En 2001, l'augmentation est de 20,5\% par rapport à 2000 et la part 
de l'éducation atteint 3,19\% du PNB, record historique aussitôt dépassé en 2002 avec $3,41 \%$.

21 Cet accroissement du budget a permis d'accueillir, entre 1999 et 2001, 2,7 millions d'étudiants supplémentaires, de financer et de réaliser plusieurs projets dont le plus important est le privilège accordé aux régions pauvres et arriérées. La mise en application du programme "Obligation scolaire » a donc été grandement accélérée. L'importance a notamment été accordée à la sécurisation des salles de classe (7,7 milliards de yuans jusqu'en 2002) et à la normalisation de la rémunération des enseignants ( 5 milliards de yuans chaque année). Dans le cadre de l'enseignement obligatoire, le budget est de 138 milliards de yuans, soit une augmentation de $88 \%$ par rapport à 1997.

Parallèlement, une structure d'aide et de soutien a été créée : 350000 étudiants bénéficient d'un prêt universitaire et les universités doivent de leur côté mobiliser chaque année $10 \%$ de leur budget pour des bourses destinées aux étudiants en difficulté. Depuis 2002, l'État attribue deux cents millions de yuans par an à des bourses de mérite destinées à aider 45000 bons étudiants en difficulté financière.

\section{Mise en œuvre des réformes}

Comment concilier la contradiction entre la demande grandissante de développement social, économique et scientifique pour l'émergence des talents et d'une main-d'œuvre de qualité et la charge écrasante d'une population à nourrir et à instruire? Pour le gouvernement chinois, la tâche est d'autant plus urgente qu'il veut accélérer la modernisation du pays. Seule la réforme peut résoudre intégralement et globalement les problèmes du développement, dans l'éducation comme ailleurs. Le gouvernement a lancé en 1998 une politique intitulée «Faire prospérer le pays par la science et l'éducation » et élaboré l'année suivante le programme "Promouvoir l'éducation au seuil du XXI siècle». Ainsi existe-t-il une approche tant théorique que pratique dans les réformes qui ont vu le jour.

Sur le plan administratif, un système de gestion dit « sectorisation et régionalisation » à deux niveaux, central et provincial, se substitue désormais à l'ancien croisement horizontal et vertical. Il a donné lieu à quelque cinq cents projets de co-construction entre le gouvernement central et les provinces, à trois cents projets de coopération inter-universitaire, au regroupement d'établissements d'enseignement supérieur (de 708 , on est passé à 302), afin de mettre fin au cloisonnement et aux doubles investissements et de laisser aux établissements une autonomie plus grande dans leur organisation administrative et pédagogique. Pour accueillir un nombre important de nouveaux étudiants, une socialisation des œuvres universitaires a été entreprise au cours de la période 2000-2002 avec des résidences d'étudiants et des restaurants universitaires, nouveaux ou réaménagés. Les travaux entrepris égalisent ou dépassent l'ensemble des travaux achevés de 1949 à 1999. En même temps, les effets du marché deviennent plus présents : les diplômés des universités n'ont plus, comme autrefois, un emploi garanti par l'État. Ils doivent désormais trouver eux-mêmes du travail.

Les affaires relevant des compétences du ministère ont diminué de $42 \%$. Ainsi, l'enseignement obligatoire dans les régions rurales, l'enseignement professionnel et la formation des adultes bénéficient d'une plus grande autonomie et sont la plupart du temps gérés par les autorités locales et les établissements eux-mêmes. 
26 Au niveau pédagogique, l'environnement s'améliore, tandis que l'éducation elle-même favorise les innovations autour d'un objectif premier : la recherche de qualité globale, afin de doter l'élève de qualités morales, créatives et pratiques. Dans l'enseignement obligatoire, un nouveau programme national a été défini, de nouveaux manuels publiés et expérimentés, de nombreux enseignants formés et/ou recyclés. Dans l'enseignement supérieur, l'examen d'entrée avec cinq matières obligatoires est remplacé par le module " $3+\mathrm{X}$ », c'est-à-dire les trois matières obligatoires au plan national (chinois, mathématiques, anglais) et une, deux, parfois trois matières laissées au choix de la province, voire de l'établissement. Les établissements sont évalués par le ministère tous les cinq ans. Le système d'unités de valeur et un cursus organisé autour de dominantes et d'optionnelles se généralise rapidement. De nouveaux manuels, y compris ceux introduits de l'étranger, sont utilisés. Un programme de 964 disciplines prioritaires, c'est-à-dire de qualité supérieure, a été lancé dans 181 établissements.

\section{Une demande croissante}

Beaucoup d'enquêtes montrent que l'investissement dans l'enseignement est le premier motif d'épargne pour les Chinois qui veulent bénéficier d'un niveau d'instruction plus élevé et d'une meilleure qualité. Des mesures adéquates visent à satisfaire ce besoin grandissant.

\section{Changer d'envergure}

La généralisation de l'enseignement obligatoire constitue le début de la modernisation de l'éducation en Chine. Cette modernisation s'observe également dans le développement nouveau et encore plus important de l'enseignement non obligatoire. Ainsi, le nombre d'élèves de l'enseignement secondaire du second cycle, déjà généralisé dans certaines villes, a doublé en 2001 par rapport à 1995. L'augmentation du recrutement des étudiants en trois ans, entre 1999 et 2002, a doublé les effectifs des universités et permis d'atteindre un taux brut de scolarisation de $15 \%$ dans le supérieur.

\section{Améliorer la qualité}

Un meilleur équipement en matériel et en personnels enseignants, dans les écoles et les collèges, une forte croissance du nombre d'élèves des bons lycées ainsi que la réorganisation du cursus universitaire sont la garantie d'une amélioration de la qualité. Les principales mesures prises sont allées dans ce sens.

\section{Promouvoir et mettre en place l'égalité et l'équité en éducation}

Trois grands programmes de soutien pour promouvoir et mettre en place l'égalité et l'équité en éducation ont été lancés par l'État dans les régions pauvres, de l'est à l'ouest, des villes à la campagne. À lui seul, le gouvernement central a attribué en 2001 cent millions de yuans en bourses d'études aux élèves de l'ouest et deux cent millions de yuans pour l'achat des manuels scolaires. Au niveau supérieur, un système de prêt universitaire et de bourses de mérite a été créé, en complément de celui qui existait déjà. 


\section{Construire un système d'apprentissage tout au long de la vie}

31 Abolition de la limite d'âge au concours d'entrée à l'université, promotion de l'autoformation et de la certification professionnelle, développement de l'enseignement en ligne et de la formation communautaire (du quartier) : autant de mesures importantes qui remportent déjà des succès.

\section{Une conception rénovée}

32 Tous les progrès mentionnés sont dus, d'une part, aux efforts de toute la société, et de l'autre, à une idéologie profondément rénovée. Ce changement peut être analysé sur deux niveaux: faire prospérer le pays par la science et l'éducation devient une politique nationale fondamentale et la place prioritaire de l'éducation est ainsi nettement définie; l'épanouissement personnel est désormais considéré comme une mission essentielle de l'éducation, sur le même plan que celle de servir la société, dont on parlait beaucoup et presque exclusivement autrefois.

33 Cet épanouissement consiste à apprendre à être, à se développer sur le plan physique, intellectuel, affectif et moral pour devenir un homme complet. En conséquence, les critères et les structures d'évaluation se modifient, les notes ont moins d'importance et les élèves sont au centre des questions scolaires. Les concepts d'éducation, de compréhension, d'apprentissage-recherche, de diversification et de personnalisation de l'éducation, ainsi que leur mise en pratique sont apparus. Construire une société d'apprentissage et créer un système de formation permanente sont devenus des mots d'ordre et l'un des objectifs de toute la société dans le domaine de l'éducation.

\section{Perspectives et difficultés}

\section{Un État arriéré et des objectifs ambitieux}

La Chine est le pays le plus peuplé du monde. Cependant, sur le plan des ressources humaines, sa situation est loin d'être satisfaisante et elle se classe parmi les pays les moins développés. Dans les exemples historiques de rattrapage des pays avancés par ceux en voie de développement, le développement des ressources humaines constitue, sans exception, une mesure prioritaire. La Chine, dont l'objectif est de parvenir au milieu du XXI siècle à une société moyennement développée, n'aura pas d'autre choix. Dans cette perspective, le gouvernement chinois s'est donné les objectifs suivants :

- créer une instance nationale pour les décisions concernant le développement des ressources humaines ;

- réorganiser les structures d'administration et de gestion en éducation en renforçant les responsabilités au niveau provincial tout en laissant au gouvernement central une fonction de macro-régulation et de contrôle ;

- pratiquer la gratuité totale de l'enseignement obligatoire ;

- diversifier les formes d'enseignement ;

- établir le système de formation pour l'emploi en accordant la priorité aux compétences ;

- réintégrer toutes les unités de recherche d'origines différentes en deux grands ensembles, entreprises et universités, en laissant à l'État un nombre de centres très restreint en complément des premiers ; 
- élever le niveau de l'internationalisation du développement des ressources humaines en renforçant les stages de perfectionnement à l'étranger et en introduisant des talents ;

- promouvoir l'informatisation de l'enseignement ;

- trouver les moyens efficaces qui permettront de créer une société d'apprentissage ;

- créer un système d'évaluation du développement des ressources humaines. 2002 , il est de 5 contre 1 . Le taux d'illettrisme, en 2000, était de respectivement 5,22\% et $11,5 \%$. Le nombre d'années d'instruction des employés non qualifiés de 10,2 dans les villes et de 6,85 à la campagne. Viennent s'y ajouter les écarts est-ouest: le taux de poursuite des études après la scolarité obligatoire (le collège), est de plus de $70 \%$ dans les six premières provinces, tandis qu'il est inférieur à $53 \%$ (la moyenne nationale) dans la moitié du pays (dix-sept provinces, dont deux ne dépassant pas $40 \%$ ). Le pourcentage des enseignants ayant les qualifications officiellement requises varie entre $50 \%$ et $95 \%$ et les régions (la campagne, l'ouest) qui en ont le plus besoin sont également celles qui en sont le plus dépourvues, en raison de conditions de vie défavorables, voire épouvantables. Vu le développement économique très rapide notamment des villes et des régions déjà bien plus riches que le reste du pays, comment équilibrer stratégie et tactiques pour assurer un progrès harmonieux dans tout le pays, avec un budget de 256,3 milliards de yuans, soit 2,87 \% du PNB ?

Les uns suivent des cours dans des classes pourvues d'équipements multimedia, bénéficient d'une salle de sport bien équipée, peuvent entrer dans des écoles privées d'élite (qui coûtent, par an, environ 60 000, 80 000, 100000 et 160000 yuans au niveau école, collège, lycée et université), lorsque les autres ont à peine de quoi écrire dans des locaux conformes aux normes de sécurité.

\section{Les conséquences de l'exode rural}

Avant 1980, la population rurale représentait plus de $80 \%$. En 2002, elle était de $67 \%$ et devrait atteindre 45 à $50 \%$ vers 2020 . Compte tenu de la croissance démographique, cela représentera trois cents millions d'urbains supplémentaires insuffisamment instruits - une charge très lourde pour l'éducation.

\section{Une créativité neutralisée par la standardisation}

L'unité et le collectivisme traditionnels, considérés non seulement comme méthodes, mais encore comme valeurs, nuisent au développement de la personnalité et de la créativité aussi bien chez les enseignants que chez les élèves. Un grand changement, même s'il est difficile, doit se produire d'abord chez les dirigeants, enseignants, parents, et ensuite chez les élèves. On est d'accord, au moins théoriquement, pour un enseignement dit « de qualité » afin de remédier aux effets néfastes d'un enseignement trop centré sur la préparation précoce aux concours. Mais devant un taux d'entrée à 
l'enseignement supérieur encore très bas et territorialement très déséquilibré (près de $70 \%$ à Beijing, $4 \%$ dans le Guizhou), ni les enseignants, ni les parents, ni même les élèves, ne peuvent ni ne veulent réellement aller dans ce sens. Résultat : un contraste paradoxal entre de bons résultats et des compétences médiocres.

\section{Pénurie d'enseignants}

Cette pénurie est à la fois qualitative et quantitative. Bien que la stabilité du statut, l'élévation de la qualification ainsi que l'augmentation de la rémunération rendent le métier plus attrayant qu'autrefois, le développement harmonieux entre les différentes régions du pays et les différentes compétences de l'élève nécessite un plus grand nombre d'enseignants aux compétences plus élevées - objectif loin d'être atteint.

\section{Retard relatif de l'enseignement secondaire du second cycle}

41 Dans une enquête de World Economic Outlook, l'écart le plus grand entre la Chine et les autres pays de développement moyen (PNB par habitant compris entre 800 et 10000 dollars) se trouve au niveau du taux d'accès à l'enseignement secondaire du second cycle. Le faire passer de $40 \%$ à $60 \%$ d'ici 2005 signifie que le nombre de lycéens passera de vingt à quarante-cinq millions, qu'il faudra doubler le nombre d'enseignants, sans compter les locaux.

\section{Inertie dans l'administration et la gestion}

Souvent, pour les Chinois, l'administration et la gestion ont la signification suivante : si l'on centralise, c'est l'uniformisation et lorsqu'on accorde plus de souplesse, c'est le désordre. Le premier est le moyen le plus simple, souvent le plus efficace et le plus bête à la fois, tandis que le second constitue, par une réaction automatique, un résultat naturel. Cela signifie qu'en Chine, au moins dans l'administration, nombreux sont ceux qui, par une mauvaise inertie héritée du passé, s'habituent déjà - sinon accueillent volontiers - à une réalité : l'anarchie dans la rigidité au lieu de la concentration issue, idéalement, d'une alliance d'autonomie et de souplesse. Cela freine la motivation, encourage la stagnation et constitue un réel obstacle pour la réalisation de tout projet, quel que soit son intérêt.

Pour mener à bien ces projets ambitieux, très importants pour l'avenir de la nation, il reste beaucoup à faire en Chine.

\section{NOTES}

1. Les 7,8 et 9 juillet jusqu'en 2002 et les 7, 8 et 9 juin à partir de 2003. 
INDEX

Mots-clés : système éducatif

Index géographique : Chine

\section{AUTEUR}

\section{KECHAO XING}

Directeur du département de français de l'institut des langues étrangères à l'université La Capitale de Beijing, chercheur à l'Institut d'éducation internationale de l'université normale de Beijing. 